PAPER

\title{
Increase in the timing coincidence of a respiration event induced by listening repeatedly to the same music track
}

\author{
Takashi G. Sato ${ }^{1, *}$, Mieko Ohsuga $^{2, \dagger}$ and Takehiro Moriya ${ }^{1, \ddagger}$ \\ ${ }^{1}$ NTT Communication Science Laboratories, NTT Corporation, \\ 3-1, Morinosato Wakamiya, Atsugi, 243-0198 Japan \\ ${ }^{2}$ Department of Robotics, Osaka Institute of Technology, \\ 5-16-1, Ohmiya, Osaka, 535-0002 Japan
}

(Received 7 October 2011, Accepted for publication 12 February 2012)

\begin{abstract}
Listening to a piece of music evokes many physiological responses including changes in respiration. The present study shows that respiration is entrained to the musical timing by comparing the changes in respiration timing and respiration period along gaining in listening experience. Participants listened to the same track once a day for 10 days. The distribution of the timings of respiration on the music track was calculated using kernel density estimation, and probability of the coincidence was evaluated statistically using surrogate data. The results show that participants unconsciously changed their respiration timing to coincide with the music track as they gain experience in listening. On the other hand, respiration period did not change.
\end{abstract}

Keywords: Breath, Entrainment, Music, Adaptation

PACS number: 43.64.+r, 43.80.+p [doi:10.1250/ast.33.255]

\section{INTRODUCTION}

There is no need to say, music is one of the popular entertainments for humans. Music has long been a central issue in various research areas. A large number of studies have revealed that listening to music is a complex phenomenon involving psychological and neurological changes [1-3]. The technical use of music to evoke changes in emotional level [4] and to support health care are also of great interest [5]. Music is a multiple bridge, indeed, between social and economic activities, between science and engineering.

One significant effect of music is related to respiration $[6,7]$. The link between respiration and music is important, because there is a strong correlation between respiration and heart rate [8-11], and also between respiration and emotion [12-16]. Revealing the relationship between music and respiration will give us the key to develop the new musical device, which will utilize respiration information in presenting music. However, the relationship between respiration response and music is not clear in some senses.

\footnotetext{
*e-mail: s.takashi@lab.ntt.co.jp

†e-mail: ohsuga@bme.oit.ac.jp

‡e-mail: moriya.takehiro@lab.ntt.co.jp
}

Previous studies showed that listening to music changes the mean respiration period and leads to coupling between the tempo and the mean respiration period [17]. These musical effects on respiration are stronger for musicians than for non-musicians [17,18]. Many researches have used respiration periods and tempo to explain the results, but for that reason there exist at least two positions to explain this phenomenon. One is that the tempo is detected and respiration periods are changed to match the tempo (tempo entrainment). The other is that musical timings like onset of phrases are detected and phase of respirations change to coincide with them (timing entrainment). The tempo entrainment gives simple explanation but the timing entrainment needs indirect explanation, that musical timings that listeners perceived are connected with tempo and timing of respiration are attracted to them, so the respiration period becomes similar to that of the tempo.

Some studies have reported that the timings of respiration the player and the listener appear to correlate [19], and respiration depths relate to the envelope of sound pressure in music [18]. According to these results, the timing entrainment supposed to be suitable. Nevertheless, the reports to directly support the timing entrainment and deny the tempo entrainment are limited. This is mainly because of the technical difficulties involved in dealing 
Table 1 List of the tracks used in the experiments. * indicates the pieces of music that used for every participants.

\begin{tabular}{ccccc}
\hline Title & Time signature & Approximate BPM & Performer & Length of time \\
\hline${ }^{*}$ Chopin: Waltz No. 6 & $3 / 4$ & 230 & Irina Mejoueva & $1: 58$ \\
${ }^{*}$ Satie: Gnossienne No. 1 & $-(2 / 2)$ & 56 & Aldo Ciccolini & $3: 01$ \\
${ }^{*}$ Lithium & $4 / 4$ & 124 & Nirvana & $4: 17$ \\
Chopin: Nocturne No. 8 & $6 / 8$ & 73 & M. J. Pires & $6: 37$ \\
Shyonen & $4 / 4$ & 77 & Mr. Children & $5: 38$ \\
Handel: Sarabande & $3 / 2$ & 59 & Academy of St Martin-in-the-Fields & $3: 26$ \\
\hline
\end{tabular}

with data in the time domain. In this paper, the term "timing of respiration" is used as timing that respiration phase changes from inspiration to expiration. The term "musical timing" is used as boundaries of musical structure that listeners perceive, and that supposed to attract the timing of respiration.

Other shortcomings of previous studies are that the effect of repeatedly listening to the same piece of music on respiration has not yet been studied and most studies compared between musicians and non-musicians but not the simple effect induced by repeatedly listening to a piece of music. In this paper, we show that listening repeatedly to the same piece of music enhances one's respiration entrainment to it. The entrainment is estimated based on the view that timings of respirations coincide statistically with musical timings. A novel technique employing surrogate data is used to estimate the entrainment.

\section{MATERIALS AND METHODS}

\subsection{Experimental Procedures}

Eight healthy persons, namely five males and three females in their twenties to fifties, participated voluntarily in this study. Since, musical effects on respiration are different between musician and non-musician [18], the difference is partly attributed to the amount of experience in music. Repeatedly listening for non-musicians is highly expected to compensate for this lack of experience and emerge the primary effect of it on respiration. So, the nonmusicians were selected as participant. A few years of private music lessons are neglected. Prior to the experiment, each participant gave informed consent after the protocol had been fully described. The participants were told that the purpose of the experiments was to observe the effect of listening to music on physiological responses.

We obtained respiratory data using an elastic chest band (Nihon-Kohden TR651-T Japan). Electrocardiograms (BIOPAC ECG100C USA) and skin blood flow (BIOPAC LDF100C USA) were also measured to avoid the participants' focusing their attention on respiration. All data were recorded at a $1 \mathrm{kHz}$ sampling rate. We tested each participant 10 times on different days but at least once per week to observe the effect of learning on respiration.
Each day, the participants sat on a sofa, and were attached sensors. The temperature, humidity, and light in the room were set at comfortable levels. After a 2 to 5 minute familiarization period, the physiological characteristics of the resting person were measured for about 1 minute to provide a baseline. The participants then listened to five pieces of music in a relaxed state without moving their bodies or tapping their fingers or feet. The pieces of music were presented in random order.

The pieces of music were chosen by the experimenter to cover the range shown in Table 1 . Three tracks were fixed and the other two were randomly chosen for each participant. This paradigm was taken to cover as many tracks as possible but to limit the total time of the experiment around 30 minute per day to ensure the participants' full concentration. One participant listened to one more track (total six tracks) in another 10 days to balance the data amount. The music was presented through a stereo speaker system (JET310.2 ELAC German).

\subsection{Data Processing}

First, the acquired relative tidal volume was realigned according to the onset of the tracks and the day of the experiment. Next, the average respiration was measured. Although the mean respiration period gives us a good insight into the data, it lacks some phase information. Thus, to measure the similarity of the respiration, we estimated the probability distribution of the timings of respirations by using a kernel density estimation [20]. The process flow is outlined in Fig. 1. The kernel distribution of the respiration event can be calculated as,

$$
p_{(t)}=\sum_{m=1}^{M} \sum_{n=1}^{N_{m}} \frac{k_{\left(t-t_{m, n}\right)}}{N_{m}}
$$

Where $k_{(x)}$ is a kernel function formed from a Gaussian kernel with a variance of $0.4 \mathrm{~s}$. Timings of respiration are the onsets of expiration as previously stated. $N_{m}$ is the number of breaths during a participant's m-th days session while listening to a certain piece of music. $t_{m, n}$ is the timings of respiration from the start of the music. Fraction $N_{m}$ is given to normalize the difference in the number of respiration cycles. 
(a)

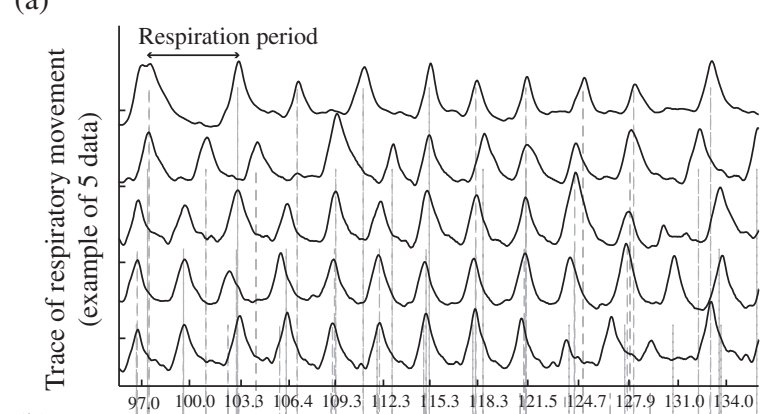

(b)

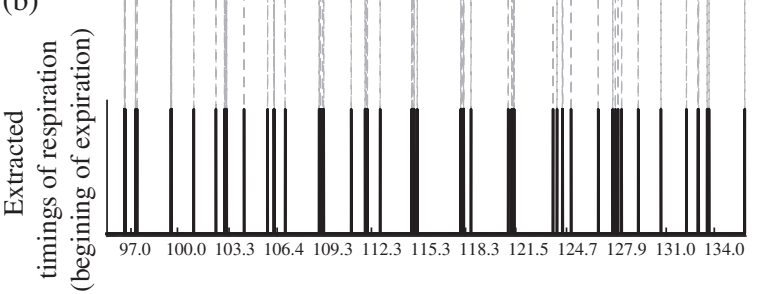

(c)

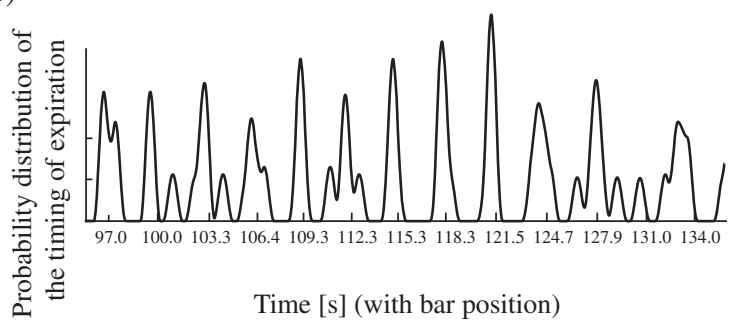

Fig. 1 Schematic of the process flow. (a): Trace of respiratory movement measured from M13 listening to Sarabande. Data from the previous 5 days are shown. The data were aligned with the beginning of the track (horizontal axes). Each division along the horizontal axis shows the position of the bar line of the track. (b): Respiratory events extracted from the respiratory trace. Onsets of expiration (local peaks in the respiratory trace) were chosen as event points (vertical dotted line). (c): Probability distribution of the respiratory event estimated by kernel density estimators. If the participants always start expiration at a specific time during the track, the probability increases. In this case, a strong coincidence, locked with the bar line, is observed at around $115[\mathrm{~s}]$ to $125[\mathrm{~s}]$.

If a person frequently takes a breath at a specific musical timing $t^{\prime}$ during the sessions, this distribution $p_{\left(t^{\prime}\right)}$ will increase. The overall coincidence of respiration at the track is a summation of power $p_{(t)}$ over $t$. We obtained

$$
G=\int_{0}^{T}\left|p_{(t)}\right|^{2} d t
$$

$G$ increases as respiration becomes coincide across the sessions. Hereafter, this parameter is named the respiratory event coincidence (REC). However, the REC is subject to parameters $T, N_{m}$, which is an undesirable parameter as regards estimating respiration coincidence. To overcome this problem and to evaluate the significance of the REC, we used a surrogate evaluation technique. An example of

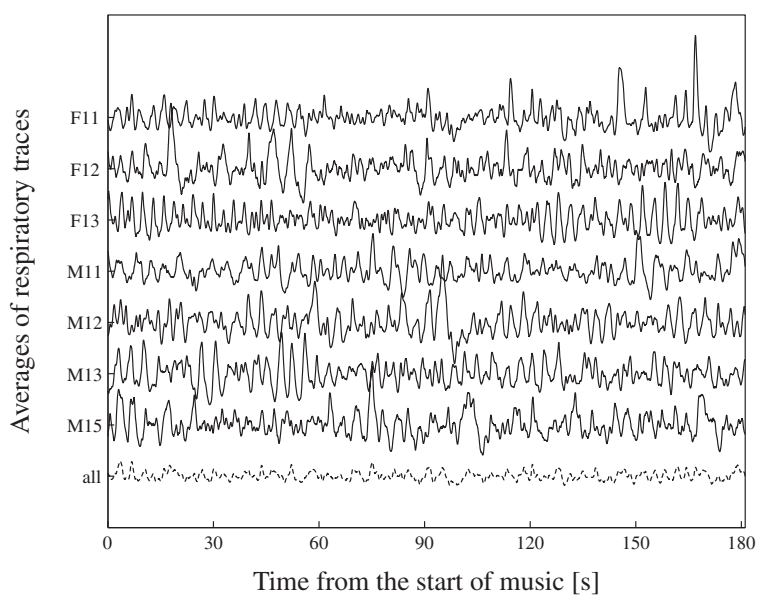

Fig. 2 The mean of respiratory curve for each participant while listening to "Gnossienne No. 1." Respiratory curves were normalized with the total power and then averaged for each participant. Data from all 10 days are used. If the participant breathed at specific timing during the music, large amplitude pattern should be observed. Note that the places where significant pattern observed are different between participants and the mean across participants (dotted line) have relatively small amplitude. However, the data is very weak to a sudden deep breath we cannot say much from this data.

evaluation techniques using surrogate data can be found in [21-23].

The surrogate data were obtained by randomizing the order of timings of respiration, preserving their intervals. Such surrogate data has a different time structure from original data but has the same $T$ and $N_{m}$ as the original data. We calculated the REC of this surrogate data, and by repeating this procedure we obtained an REC histogram. The probability of measuring the original REC is estimated from complementary cumulative distribution of the histogram. Its statistical significance between groups was tested using ANOVA.

\section{RESULTS}

The recorded data were down sampled to $100 \mathrm{~Hz}$ and processed in the later analysis using Matlab. Figures 1(a) and 2 shows an example of the recorded data. The expiration onset was extracted (Fig. 1(b)) and the respiration periods were calculated. Some of the participants reported that they already knew or had listened to some of the tracks (one third of the cases), but they had not listened to them frequently or memorized whole phrases. One participant retired because we could not conduct a next experiment within a week due to the schedule conflict.

The presentation of music changed the respiration rate significantly compared with the baseline in one third of the cases (paired $t$-test $P<0.05$ in Fig. 3). As previous studies have observed [17,24], the music reduced the mean 


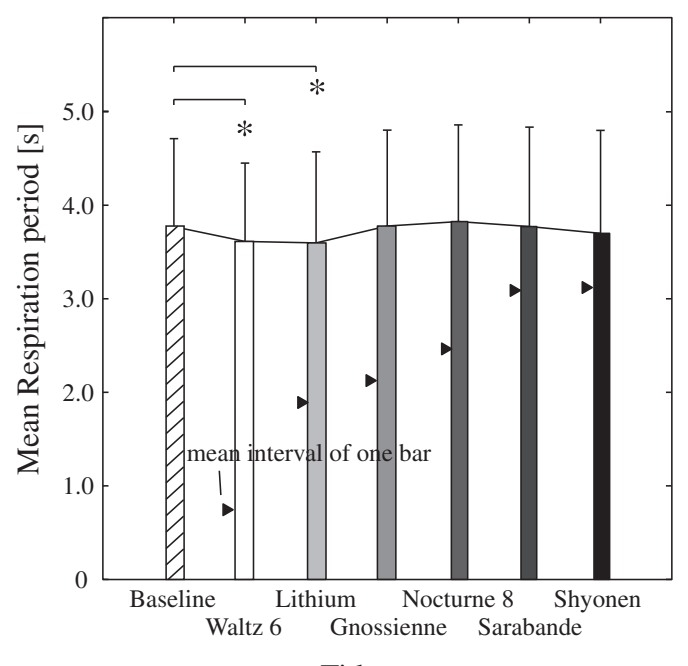

Title name

Fig. 3 Mean respiration periods for each participant listening to different tracks. The averages were calculated using all 10 days' data. The tracks are aligned from faster tempo to slower tempo. The bar lengths are displayed instead of tempi to match the scale. The respiration period decreased for the fast tempo ${ }^{*} p<0.05$ versus baseline. There were no significant correlations between tempo and mean respiration period.

respiration period. However, there was no obvious correlation between tempo and mean respiration period.

The probability densities of the expiration onsets were calculated using the kernel density estimation method (Fig. 1(c)). The probability densities had many peaks and in some cases the peaks correlated with the bar line, however, the correlation always lasted only a few seconds. The RECs were calculated from the probability density, and their significance were evaluated using surrogate data. Figure 4 shows an example REC histogram.

\subsection{Learning Effect}

To analyze the effect of repeatedly listening to the same piece of music, 10 days' data were divided into three groups; data obtained from the 2nd to 4th day, the 5th to 7th day, and the 8th to 10th day. Data from first day were excluded to balance the data amount, thus each group consisted of three days' data from participants. Hereafter, we refer to the group as experience period because it reflects the increasing experience of participants. The changes in the mean respiration periods are shown in Fig. 5. Music presentation always shortened the mean respiration period, however, no significant difference was observed with respect to the experience period. A slight correlation with tempo was observed.

The probability distribution was then calculated from the extracted respiration event. The variance of the kernel used in the kernel density estimation is allowable regarding

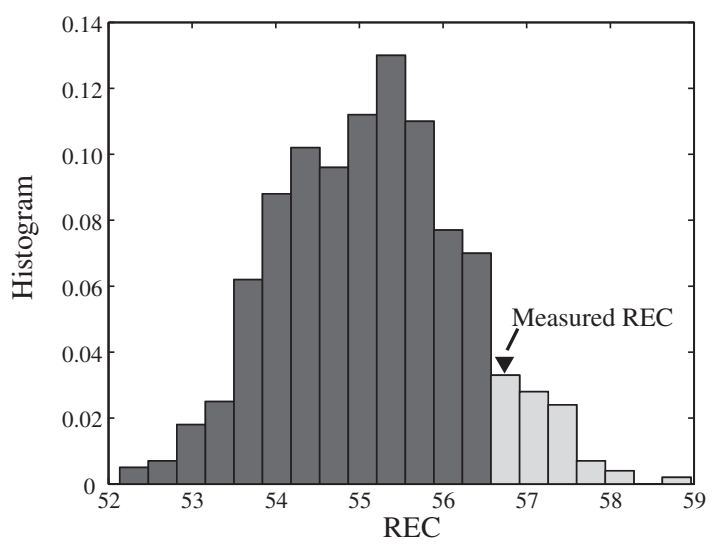

Fig. 4 Example of the histogram of surrogate RECs. Surrogate RECs were calculated from surrogate data and used to construct the histogram. The last three days' data (day 8th to 10th) from all the participants who listened to Sarabande were used. The triangle shows the REC of the measured data. The probability of obtaining the measured REC from the histogram can be estimated from the complementary cumulative distribution of the histogram, which is same as the proportions of the light gray area.

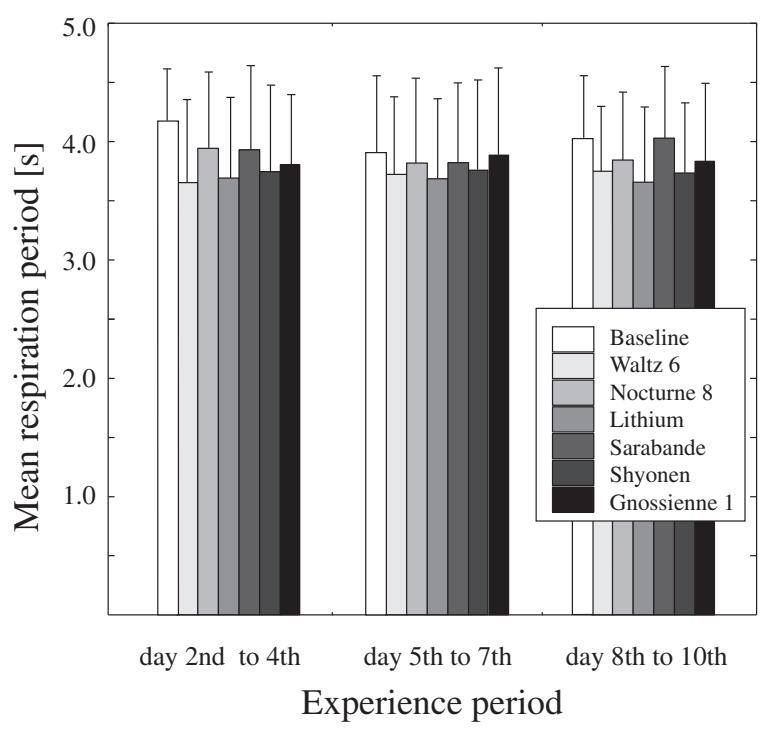

Fig. 5 Change in mean respiration period. Nine data (first of 10 data excluded) recorded from all participants are divided into three groups according to the experience day. The averages are for all participants for each track. A slight change was observed in relation to the change in experience period.

the preposition that a person can consciously control his respiration cycle [25]. While it was not obvious whether the peaks were the result of entrainment or simply a random occurrence, the REC probability was estimated as described in section 2.2. The REC probability shows a clear decline as the participants gain more experience (Fig. 6). A significant difference (ANOVA test, $p<0.05$ ) was obtained with respect to the experience periods. 


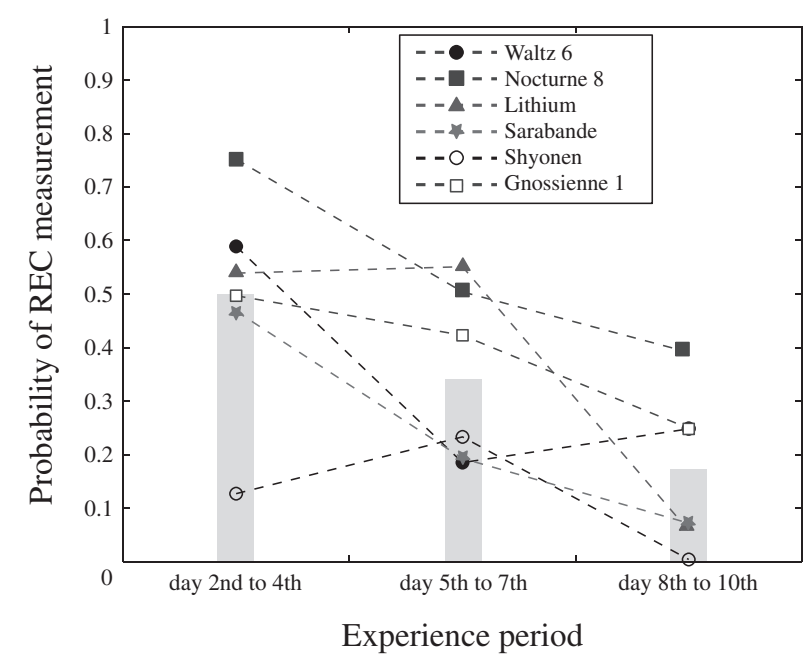

Fig. 6 Probability of REC measurement. Nine data (first of 10 data excluded) recorded for all participants were divided into three groups according to the experience day. The strength of coincidence was calculated using Eq. (2) from the probability distribution (Fig. 1(c)) and the probability of achieving such strengths were estimated using surrogate data (Fig. 4). An increase in experience resulted in a lower probability, which means the coincidence of the respiratory event has increased. An ANOVA test revealed a $p<0.05$ significance.

\section{DISCUSSION}

\subsection{Advantages and Disadvantages of Using REC}

There are several advantages of using the REC. The first is that it is independent of respiration depth. The obtained data usually include both shallow and deep respirations, and because respiratory traces are non-linear, spectral analysis can often be fooled by a sudden large change in respiration depth. But with our method, respiration is treated as a time event so the above problem does not arise.

Treating respiration as time events gives us another advantage, namely we can achieve high resolution in a time over frequency-based analysis. The REC time resolution depends only on the width of the Gaussian kernel, so our method can deal precisely with the coincidence of a respiratory event. However, what respiration event should be selected as respiratory marker is unclear.

The REC itself is very sensitive to data length and number of respirations. To overcome this problem the surrogating technique was used. Since the REC is the integral over time, REC evaluation with surrogates shows the statistical significance of entrainment. This is advantage in sense that musical timing is not necessary to be defined but disadvantage is that musical timing the place where entrainment occur become unclear.

In previous studies, bar points are used to evaluate respirations $[17,19]$. However, bar points are not the only musical timings that work as attractor of respiration. They may be starts of the vocal or sudden silences preceding the next phrase. Outlining these musical timings are going to be difficult task. The big advantage of REC evaluation is that strength of the music influenced the respiration can be estimated without defining these musical timings.

\subsection{Learning Effect}

Previous studies had reported the difference between the respiration period of musicians and non-musicians. However, nothing has been reported about whether or not we need special training to achieve such synchronization. One of the major differences between musician and nonmusician is amount of experiences in music. If entrainment of respiration to the music relates to this, non-musicians are expected to change their respiration pattern as they gain experience. In our study, repeatedly listening to the same track reduced the REC probability (Fig. 6). This means that coincidence of respiration increases as gaining experience. In other words, the participants somehow learned to change their respiration with pieces of music, despite simply and passively listening to them. On the other hand, little change was observed in respiration period with regard to experience period (Fig. 5). Further discussion about the difference is done in next section.

\subsection{Tempo Entrainment or Timing Entrainment}

As previously stated, there are at least two positions to explain the change in respiration, namely tempo entrainment and timing entrainment.

Most previous studies assume that tempo plays a critical role in the entrainment of respiration, and have tried to show that effect by using respiration period as an index for its evaluation $[17,26]$. Despite a significant change in respiration period compared to the baseline, little correlation between tempo and respiration period was observed. The result is comparable with previous research at this point, because they reported a significant correlation in musicians but not in non-musicians, and in our experiment the participants were not musicians.

But if the tempo entrainment is important, a change in the respiration period, which is also an index of time length, should appear as an effect of gaining experience. Gradual shift in the mean respiration period towards tempo or, at least, a reduction in the variations of periods, was expected with regard to experience periods. However, the mean respiration period exhibited no noticeable change. In contrast, the REC probability decreased. As discussed in previous section 4.1, the change in REC implies the existence of musical timings, which attract timing of respiration. Our result showed that the priority of the timing entrainment is higher than tempo entrainment. 
To the best of our knowledge, this study is the first to observe the coincidence probability of respiration with regard to increased experience. The result suggests the musical timing entrain respiration. Then, what is the musical timing? Answer is still not clear for us, but some discussion can be made. The notion appears to be closely related to the perception of a rhythmical pattern. Theoretical analysis suggests that the critical feature of a rhythm is the onset sequence of its notes [27]. By repeatedly listening to the same piece of music, we are unconsciously searching for such onsets and learning which onset should be tracked. These selected onsets may compose phrases and become musical timings that entrain respiration. Figure 2 suggests that there likely exist individual differences of such musical timings. Although this musical timing is not necessary combined with tempo, music with fast tempo is supposed to have many of them. The reason why relationship between tempo and respiration period has observed in previous studies may attribute to this.

\subsection{Limitation}

The number of participants was relatively small. However, it should be noted that the total amount of data was larger than that of previous studies due to the repeated recordings, and this is why we think we had sufficient data to draw a conclusion. Data of some tracks consist of different participants. Individual differences in attracted musical timing, which is suggested by Fig. 2 may affect the result. However, the advantage of the REC analysis that need not to explicitly define musical timings works to suppress such individual differences and most results showed same trend in Fig. 6. Thus, we think individual differences had little effect on our analysis. Only a few pieces of music were examined and the choice depended strongly on the experimenter, although this is a common problem in the music research field. Evaluation of REC is rather new technique to estimate the coincidences; we expect further reports to testify its reliability.

\section{CONCLUSION}

This study has showed that repeatedly listening to the same track increases the probability that timing of respiration occur, at a specific musical timing on the track. Our novel method made this evaluation possible. The method estimated the coincidence from the distribution of the timing of respiration, and statistically evaluated its coincidence probability using surrogate data. The result shows that participants unconsciously learned to change their respiration and it implies the existence of musical timing that attracts timing of respiration. Since, the entrainment to tempo is not observed as a primary effect of learning, it is shown that entrainment of respiration to music is caused by timing entrainment but not by tempo entrainment.

\section{ACKNOWLEDGMENTS}

We are grateful to Yuuki Ooishi (NTT Communication Science Labs.) for his kind advice and comments. All experimental procedures were approved by the NTT Ethics Committee.

\section{REFERENCES}

[1] C. M. Diserens, "Reactions to musical stimuli," Psychol. Bull., 20, 173-199 (1923).

[2] S. Koelsch and W. A. Siebel, "Towards a neural basis of music perception,” Trends. Cogn. Sci., 9, 578-584 (2005).

[3] L. Trainor, "The emotional origins of music," Phys. Life Rev., 7, 44-45 (2010).

[4] I. Peretz and R. Zatorre, The Cognitive Neuroscience of Music (Oxford University Press, London, 2001).

[5] G. Bernatzky, P. Bernatzky, H. Hesse, W. Staffen and G. Ladurner, "Stimulating music increases motor coordination in patients afflicted with Morbus Parkinson," Neurosci. Lett., 361, 4-8 (2004).

[6] P. Gomez and B. Danuser, "Affective and physiological responses to environmental noises and music," Int. J. Psychophysiol., 53, 91-103 (2004).

[7] M. Mockel, L. Rocker, T. Storks, J. Vollert, O. Danne, H. Eichstodt, R. Moller and H. Hochreins, "Immediate physiological responses of healthy volunteers to different types of music: cardiovascular, hormonal and mental changes," Eur. J. Appl. Physiol., 68, 451-459 (1994).

[8] D. L. EckBerg, "The human respiratory gate," J. Physiol., 548, 339-352 (2003).

[9] O. Gilad, C. A. Swenne, L. R. Davrath and S. Akselrod, "Phase-averaged characterization of respiratory sinus arrhythmia pattern," Am. J. Physiol. Heart Circ. Physiol., 288, H504H510 (2005).

[10] B. T. Haymet and D. I. McCloskey, "Baroreceptor and chemoreceptor influences on heart rate during the respira-tory cycle in the dog," J. Physiol., 245, 699-712 (1975).

[11] C. Schafer, M. G. Rosenblum, J. Kurths and H. H. Abel, "Heartbeat synchronized with ventilation," Nature, 392, 239240 (1998).

[12] F. A. Boiten, "The effects of emotional behaviour on components of the respiratory cycle," Biol. Psychol., 49, 2951 (1998).

[13] P. Gomez, W. A. Stahel and B. Danuser, "Respiratory responses during affective picture viewing," Biol. Psychol., 67, 359-373 (2004).

[14] I. Homma and Y. Masaoka, "Breathing rhythms and emotions," Exp. Physiol., 93, 1011-1021 (2008).

[15] Y. Masaoka and I. Homma, "The source generator of respiratory related anxiety potential in the human brain," Neurosci. Lett., 283, 21-24 (2000).

[16] C. J. E. Wientjes, "Respiration in psychophysiology: methods and applications," Biol. Psychol., 34, 179-203 (1992).

[17] F. Haas, S. Distenfeld and K. Axen, "Effects of perceived musical rhythm on respiratory pattern," J. Appl. Physiol., 61, 1185-1191 (1986).

[18] L. Bernardi, C. Porta, G. Casucci, R. Balsamo, N. F. Bernardi, R. Fogari and P. Sleightet, "Dynamic interactions between musical, cardiovascular, and cerebral rhythms in humans," Circulation, 119, 3171-3180 (2009).

[19] T. Yamamoto, N. Fujii and Y. Miyake, "A musical communication between a player and a listener," Proc. IEEE Int. Symp. CIRA, pp. 330-335 (2003). 
[20] C. M. Bishop, Pattern Recognition and Machine Learning (Springer, Berlin, 2006).

[21] Y. Kusunoki, M. Fukumoto and T. Nagashima, "A statistical method of detecting synchronization for cardio music synchrogram," IEICE Trans. Fundam., E86-A, 2241-2247 (2003).

[22] H. Seidel, H. Herzel and D. L. Eckberg, "Phase dependencies of the human baroreceptor reflex," Am. J. Physiol., 272, H2040-H2053 (1997).

[23] H. Seidel and H. Herzel, "Analyzing entrainment of heartbeat and respiration with surrogates," IEEE Eng. Med. Biol. Mag., 17 (6), pp. 54-57 (1998).
[24] D. S. Ellis and G. Brighouse, "Effects of music on respiration and heart-rate," Am. J. Psychol., 65, 39-47 (1952).

[25] G. F. Rafferty and W. N. Gardner, "Control of the respiratory cycle in conscious humans," J. Appl. Physiol., 81, 1744-1753 (1996).

[26] L. Bernardi, C. Porta and P. Sleight, "Cardiovascular, cerebrovascular and respiratory changes induced by different types of music in musicians and non-musicians: the importance of silence," Heart, 92, 445-452 (2006).

[27] D. J. Povel, "A theoretical framework for rhythm perception," Psychol. Res., 45, 315-337 (1984). 